\title{
Hedging in Popular Scientific Articles on Medicine
}

\author{
Csongor Alexandra, Rébék-Nagy G \\ Department of Languages for Specific Purposes, Faculty of Medicine, University of Pécs, Pécs, Hungary
}

Introduction: The aim of this study is to investigate the process of rewriting medical research papers for the lay public. The latest findings of medical research often appear in the popular media. It is interesting to see what happens to a scientific text when it is transmitted to a new audience. Hedging is usually interpreted as a characteristic feature of scientific discourse. This study focuses on hedging, which also tends to be applied in popularized articles in the field of medicine.

Material and method: Five medical research articles on prenatal vitamins and their online popularizations were examined by means of a text analyzing software, focusing on lexical items considered as hedges. The frequency and the overall percentage of hedging devices with respect to the total number of words were recorded in the five popularizations.

Results: The results of the present study suggest that the linguistic strategy of hedging is applied in popular articles. Approximators, auxiliaries, epistemic verbs and adverbs expressing tentativeness, possibility and politeness were used in the corpus. The overall percentage of the lexical items commonly regarded as hedges, with respect to the total number of words, was $1-2.2 \%$ in the five articles. The writers also use linguistic techniques that can be interpreted as attribution shields. These defense tools convey the meaning that it is the researcher, rather than the writer, who is responsible for the truth of the information.

Conclusions: Hedging as a means of uncertainty and negative politeness technique is used in the popularizations analyzed. The present study should be extended to investigate tendencies in popularization of scientific information.

Keywords: scientific popularization, medical research article, hedging, politeness

Received: 7 May 2012

\section{Introduction}

The aim of this study is to show how scientific knowledge is transmitted to the lay public, focusing on the language of popularized medical research articles. The internet is an important source of information about medical advances in the field of health, especially for lay people. Online journals routinely use the general medical magazines to obtain information on the latest findings of medical research [1]. The news media translates the content of scientific articles and influences the decision making process of the audience. It is also suggested by investigators that the popular media amplifies the transmission of medical information from the scientific literature to the researchers. It appears that popularization has an effect on the scientific audience, and it also enhances the information flow of the scientific literature [2]. This information enhancement is even more justified in the new era of the World Wide Web.

The aim is to shed light on some aspects of how scientific articles are transmitted to the popular press. The most important elements of the study are the examination of the reader-writer configuration in the selected texts and also the role of hedging in the different genres as it reflects the relation of the readers and the writer. Hedging is a characteristic feature of medical research articles [3,4]. It indicates tentativeness, possibility and politeness by using different linguistic devices. The question is whether this tool

Correspondence to: Alexandra Csongor

E-mail: alexandra.csongor@aok.pte.hu is used in popularized communication of research findings for non-specialist readers.

\section{Material and method}

The corpus of this study includes five popularizations of medical research articles from different medical journals. The articles were selected so that prenatal vitamins, a typical area of medicine was covered. The popular articles were selected with the help of internet search engines. The simple search methods were not satisfactory, so advanced search techniques and other tools for managing health information were applied such as the use of WebMD, OmniMedicalSearch, Pubmed, and HealthMash. The current study is limited to the differences in the reader-writer relationship of the two text types, and also on some effects that this particular configuration has on the discourse elements. A manual analysis and a software analysis with Textanz text analyser were carried out. The selected lexical items commonly regarded as hedges were classified into different categories and their frequency and overall percentage were recorded.

\section{Results}

The Medical Research Articles (MRA) chosen from prestigious medical journals provide information about scientific findings on maternal vitamins and nutrition. In the scientific articles the author is the researcher, who is writing about his or her own findings. The readers are usually other researchers, either of the same field of study or from broader spheres of science. In popularized genres there may 
Table I. The frequency and overall percentage of the different types of hedges in the five popularized articles

\begin{tabular}{|c|c|c|c|c|c|}
\hline Types of hedges & $\begin{array}{c}\text { Frequency } \\
\text { Text1 }\end{array}$ & $\begin{array}{c}\text { Frequency } \\
\text { Text } 2\end{array}$ & $\begin{array}{c}\text { Frequency } \\
\text { Text } 3\end{array}$ & $\begin{array}{c}\text { Frequency } \\
\text { Text } 4\end{array}$ & $\begin{array}{c}\text { Frequency } \\
\text { Text } 5\end{array}$ \\
\hline Auxiliaries (may, might) & 3 & 2 & 1 & 1 & 1 \\
\hline Epistemic verbs (suggest, tend) & 0 & 1 & 0 & 0 & 3 \\
\hline Adverbial phrase (likely to) & 1 & 1 & 2 & 0 & 1 \\
\hline Numerical hedges (often, almost, about, sometimes, rarely, most) & 2 & 2 & 3 & 3 & 5 \\
\hline Percentage of hedges with respect to total number of words & $2.2 \%$ & $2.2 \%$ & $1 \%$ & $1.2 \%$ & $1.4 \%$ \\
\hline
\end{tabular}

be other configurations of readers and writers. The popular articles in the corpus have the same topics but not the same authors as the scientific text that they aim to review. The texts selected were published in the health or science section of online magazines, such as the New York Times. The writers are usually science journalists, who transmit scientific knowledge to lay people. The reader and writer configuration is modified in these popularizations. The writers in this corpus are non-specialists, and as opposed to the MRA-s, the target audience is the general public. The non-specialist writer is transmitting the results of a specialist to an unknown group of mostly non-specialist readers.

Differences in the reader-writer relationship and the special communication medium of the internet have an effect on the language use of the texts. The question of the study is what linguistic features characterize these online publications and how the original article is transmitted to the new communicational context. First of all, the popular scientific texts were much shorter than the original articles. The details of the study are not important in this genre, in most cases the materials, results and the conclusions were summarized. Popularizations usually started with a remarkable assertion or the conclusion of the study to capture the attention of the reader. While in the scientific articles rigid conventions were followed, in the popular articles a more varied vocabulary was used. The technical terminology was often avoided, or more comprehensible synonyms were used. The titles of the articles were also shortened and contained only key words in accordance with the genre of news and the new communication medium of the internet. The main communicative function was to assure the reader that the text was interesting and current.

Another important aspect of the study was to compare the linguistic strategy of hedging in the two different genres. The most frequent lexical items commonly regarded as hedges were examined in the five online articles by means of a text analyzing software. Table I. shows the frequency, types and percentage of hedges in the five popularized texts.

The overall percentage of the lexical items commonly regarded as hedges with respect to the total number of words was $1-2.2 \%$ in the five articles. In addition to the above hedges, the writers used linguistic techniques to ascertain that the readers cannot question the information provided. They were interpreted as hedges, as their aim was to limit the responsibility of the writer for the information in the articles. The communicative function was to make the readers believe that the information was based on credible scientific data. Linguistic devices, such as the ones in the following list were used in minimum 4 and maximum 12 cases in the popularizations analyzed:

"a new study has found"
"the scientists found that"
"researchers have determined that"
"the authors of the new report say"
"says the study"
"a study has revealed" etc.

\section{Discussions}

Scientific popularization has various levels and it has already been studied by linguistic experts $[3,5,6]$. The online articles are useful means of communication between scientists and lay people, however in most cases these are not written by the researchers themselves. In the articles investigated the writer is a non-specialist, who transmits the information of science to the lay public. The writer is placed between the researchers and the readers [3]. This special configuration has an effect on how the findings of the scientific articles are interpreted.

The main communicative function in popularizations is to capture the readers' attention. The popularized articles are much shorter than the MRA-s, and they are adapted to the needs of the new communication medium. There are only key words in the titles of the articles and their main task is to convey the importance and relevance of the topic to the readers [7]. To sum up, popularizations are different from MRA-s in two essential aspects. They have a different target audience and different authors.

According to earlier studies hedges are central elements of scientific communication, especially of MRAs [3,4]. However there are conflicting ideas about hedging in popularizations [5]. One point of view claims that the tentative tone of the research papers is changed, and only definite assertions are used in popular articles [7]. As a result, hedging is not required and it is rarely or not used in popularized texts. However, the results of the present study suggest that the linguistic strategy of hedging tends to be applied in popular articles. Numerical hedges, auxiliaries, epistemic verbs and adverbs interpreted as hedges were found in the corpus. Moreover, the notion of hedging was broadened in the articles investigated. Expressions like the study has found, 
or scientists revealed etc. are repeated throughout the texts. These may be interpreted as defense tools against the criticism of the audience, and also convey the meaning that it is the researchers and not the writer of the popularized version that are responsible for the truth of the information. In this sense they are used to reduce the writer's responsibility. They may act as attribution shields, which attribute the claim to someone. However, it is difficult to distinguish whether they are used as hedges or simply reporting verbs [8].

\section{Conclusions}

The results of science are not only read by the researchers' community but they are important for the lay people as well. There are different reader-writer configurations in the genre of medical research articles and popularizations. The special relationship of the authors and the audience- together with other communication factorsinfluences the linguistic strategies used. The study of popular scientific texts has often produced controversial results concerning the use of hedging. The results suggest that hedging as a means of uncertainty and negative politeness technique is used in the popularizations ana- lyzed. To sum up, it may suggest that science journalists use the linguistic devices of scientific communication in order to protect themselves and also to write reviews that appear to be more scientific. The present study should be extended to examine thoroughly the tendencies used in popularizations.

\section{References}

1. Entwistle V. Reporting research in medical journals and newspapers. BMJ 1995;310:920-923.

2. Phillips DP, Kanter EJ, Bednarczyk B, Tastad PL. Importance of the lay press in the transmission of medical knowledge to the scientific community. N Engl J Med 1991;17(325):1180-1183.

3. Myers G. The Pragmatics of Politeness in Scientific Articles. Applied Linguistics. 1989;10:1-35.

4. Rébék-Nagy G. Modulation of Authors' Claims in Medical Research Articles. PhD thesis, 2000

5. Varantola K. Popularization strategies and text functional shifts in scientific/ technical writing. LSP Newsletter. 1987;10:33-52.

6. Varttala T. Remarks on the Communicative Functions of Hedging in Popular Scientific and Specialist Research Articles on Medicine. English for Specific Purposes. 1999;18:177-200.

7. Smith DE. The process of popularization-rewriting medical research papers for the layman. JR Soc Med. 1987;80(10):634-636.

8. Crompton P. Hedging in academic writing: some theoretical problems. English for Specific Purposes. 1997;16:271-287. 